\title{
Entanglement in fermionic systems at a quantum phase transition
}

\author{
Henrik Johannesson ${ }^{1}$ and Daniel Larsson ${ }^{2}$ \\ ${ }^{1}$ Department of Physics, Guteborg University, Guteborg SE-412 96, Sweden \\ ${ }^{2}$ School of Physics and Astronomy, University of Birmingham, Birmingham B15 2TT, United Kingdom
}

Received April 16, 2007

\begin{abstract}
We consider recent results on the use of the single-site entanglement measure for identifying and characterizing a quantum phase transition in systems of interacting fermions. We discuss the extension of these results to fermionic models where the single-site entanglement may fail to signal a quantum phase transition, with particular attention given to the one-dimensional extended $U V$ Hubbard model.
\end{abstract}

PACS: 71.10.Fd Lattice fermion models (Hubbard model, etc.);

03.65.Ud Entanglement and quantum nonlocality (e.g. EPR paradox, Bell's inequalities, GHZ states, etc.);

03.67.Mn Entanglement production, characterization, and manipulation; 05.70.Jk Critical point phenomena.

Keywords: fermionic models, quantum phase transition, Hubbard model.

\section{Introduction}

In the last few years there has been a growing interest in quantum many-particle systems from the point of view of quantum information [1]. The interest is driven by the need to go beyond the present understanding of how to operate a few qubits and build scalable and fault-tolerant devices that can be easily controlled and manipulated. New ideas for carrying out quantum information tasks, such as quantum state transfer, have also increased the need to better understand the behavior of many-particle systems. These goals have led to a vivid exchange of ideas between the quantum information and condensed matter communities. As a result, novel approaches inspired by quantum information theory are now actively being pursued for attacking problems in condensed matter physics, in particular in the field of strongly correlated electrons. Conversely, analytical and numerical methods - as well as intuition and «know-how» - from condensed matter physics naturally find their way into quantum information science when exploring various designs of solid-state hardware for quantum information processing.

One of the central concepts of quantum information theory [2] is that of entanglement: A state of a composite system is said to be entangled if it cannot be written as a direct product of the individual states of its constituents. As pointed out by Schrцdinger in his famous «cat paradox» paper from 1935, entanglement (in German «Verschrдnkung») lies at the very heart of quantum mechanics: «... [the fact that] the best possible knowledge of a whole does not necessarily include the same for its parts. [...] The whole is in a definite state, the parts taken individually are not... [This is] not one, but the essential trait of the new theory, the one which forces a complete departure from all classical concepts» [3]. Today we understand that entanglement - and the non-local correlations that go with it [4] - are not only intrinsic to the fabric of reality, but can also be exploited as a resource for processing quantum information. Much of current theoretical research aims at quantifying «how much» entanglement there is in a given quantum many-particle system and how it is distributed over the system. This is a prerequisite for identifying useful Hamiltonians to produce and control entangled states, and also for exploring schemes for quantum computing which rely on the entanglement of a large number of degrees of freedom (such as topological quantum computing [5] or «one-way» quantum computing [6]). There is also a fundamental aspect to this endeavor: By studying entanglement properties of a many-particle system one may extract information about 
complex ground state wave functions without having to calculate them explicitly!

One particular circle of problems that has attracted attention in this context center around the use of entanglement measures for identifying and characterizing quantum phase transitions, following the pioneering work by Osterloh et al. [7] and Osborne and Nielsen [8]. A quantum phase transition (QPT) happens when the zero-temperature quantum fluctuations in a quantum many-particle system cause a transition from one type of ground state to another [9]. Such transitions are induced by the change of a physical parameter (magnetic field, chemical potential, pressure, ...) that enhances the quantum fluctuations, or their effect on the system. The study of QPTs has today become an important theme in condensed matter physics. The reason is that the very existence of a quantum critical point, i.e. the point in the phase diagram at which the QPT takes place, influences the physical properties of the system also at experimentally accessible temperatures, and opens up a quantum critical regime with characteristics very different from what is expected from conventional «text-book» theory. Theoretical scenarios invoking the existence of a (hypothetical) QPT to explain certain anomalous, so called «non-Fermi liquid» properties of a system are actively pursued in the field of heavy-fermion physics [10]. Similar attempts are being launched also at other problems in the physics of strongly correlated quantum matter, from the study of complex oxides [11] to ultra-cold gases trapped in optical lattices [12].

The change of a ground state at a QPT is generically associated with a non-analyticity in the ground state energy [9]. This is most often associated with an avoided level crossing, where the non-analyticity develops asymptotically in the thermodynamic limit. A special class of QPTs are those where the transition is driven by a field that couples to an operator that commutes with the rest of the Hamiltonian. In such a case the non-analyticity simply reflects the level crossing that goes with the transition. Barring accidental cancellations, a nonanalyticity in the energy automatically propagates into the elements of the density matrix of the system. Since any measure of entanglement [13] is constructed from a (reduced) density matrix one expects that the non-analyticity will somehow show up also in the ground state entanglement. But how exactly does it show up? And moreover, how does the scale invariance at a continuous QPT (as happens when there is an avoided level crossing, and the Hamiltonian supports only local, or quasi-local, interactions) manifest itself in the scaling of the entanglement as one approaches the quantum critical point?

These are important questions, which are now just beginning to be tackled. Some answers can already be drawn from the large body of results for spin-1/2 models in one dimension (interacting qubits on a 1D lattice). For example, based on results for critical spin-1/2 chains, $\mathrm{Wu}$ et al. [14] conjectured that a discontinuity [divergence] in the [derivative of the] ground state concurrence is associated with a first [second] order QPT, barring the appearance of accidental singularities [15]. The subsequent proof that any entanglement measure can be expanded as a unique functional of the first derivatives of the ground state energy (with respect to the parameters that control the QPT) puts this intuition on firm ground [16], as does results from standard scaling theory [17]. Other related results, employing the notion of localizable entanglement [18], entanglement entropy [19], and generalized global entanglement [20] have also been obtained. As to the property of scale invariance at a continuous QPT, this is strikingly seen in in the logarithmic divergence of the block entanglement entropy with the length of the block [21-23]. While some important issues remain to be clarified - in particular about the connection between the non-local correlations implied by entanglement and the long-range («classical») correlations emerging at a QPT [24] — the basic features of entanglement properties of critical spin-1/2 systems are by now fairly well understood. The frontier of this research area has advanced rapidly in the last year and is now making contact with potential applications in quantum information science, an example being the use of spin chains for quantum state transfer $[25,26]$.

In contrast, less is known about the details of the entanglement - QPT connection for systems of itinerant particles. One difference from lattice qubit systems is that the requirement of [anti-]symmetrization of the wave function for indistinguishable [fermions] bosons implies a physical Hilbert space that lacks a direct product structure. It is then no longer obvious how to define the very notion of entanglement, i.e. the property that a many-particle wave function does not factorize into a product of single-particle functions. There is an ongoing debate how to unambiguously resolve this issue [27]. One possible way to circumvent the problem and recover a direct product structure of the Hilbert space, was suggested by Zanardi [28]: Passing to an occupation number representation of local fermionic modes one takes as basis the $4^{L}$ states $|n\rangle_{1} \otimes|n\rangle_{2} \otimes \ldots \otimes|n\rangle_{L}$, where, for spin-1/2 fermions, $|n\rangle_{j}=|0\rangle_{j},|\uparrow\rangle_{j},|\downarrow\rangle_{j}$, or $|\uparrow \downarrow\rangle_{j}$ is a local state at site $j$, with $L$ the number of sites on the lattice. Note that the local Hilbert spaces are attached not to the individual particles, but to the distinguishable sites of a lattice. Also note that the labeling of occupied states by the spins of the particles is not unique. Indeed, one could have opted instead for a labeling in terms of the (crystal) momenta of the particles. As this would imply infinite-dimensional local Hilbert spaces, one usually makes the simpler choice with spin quantum numbers as labels. Needless to 
say, which local Hilbert space to pick is dictated by the (Gedanken) experiment that is to be carried out: The states that span the local Hilbert spaces are those which diagonalize the operator that represents the observable that is to be measured. Still, even with the simplest choice of spin labeling, the problem is harder than for qubits since now each lattice site is associated with four local states instead of two. For pure states the entanglement (von Neumnann) entropy remains a well-defined measure of entanglement, but for mixed states one has to trade the preferred concurrence measure (related to the entanglement of formation) [29] for the less tractable measure of negativity [30].

Entanglement behavior at a fermionic QPT Leaving the difficult problem of mixed states aside, we shall here focus on the use of the pure state entanglement entropy as a marker of QPTs in systems of interacting spin-1/2 fermions [31]. For that purpose we split our lattice fermion system into two parts, $A$ and $B$, and define as usual the entanglement entropy $\mathcal{E}$ of the ground state $\left|\psi_{0}\right\rangle$ (with respect to the chosen partition) by [32]

$$
\mathcal{E}=-\operatorname{Tr}\left(\rho_{A} \log _{2} \rho_{A}\right) .
$$

The reduced density matrix $\rho_{A}$ is obtained from the full density matrix $\rho=\left|\psi_{0}\right\rangle\left\langle\psi_{0}\right|$ by tracing over the local states belonging to $B: \rho_{A}=\operatorname{Tr}_{B}(\rho)$. (Taking a trace over the local states belonging to $A$ gives the same result.) By choosing $A$ to be a single site, call it $j$, with $B$ the rest of the system, one thus arrives at the single-site entanglement. Assuming translational invariance, and that the ground state $\left|\psi_{0}\right\rangle$ is a superposition of basis states with the same number of particles and with the same total spin (as guaranteed by a translational invariant Hamiltonian that conserves total spin and particle number), it is easy to verify that the reduced ground state density matrix $\rho_{j}$ for the single site $j$ is diagonal in the chosen basis. Introducing the expectation values that a single site is doubly occupied $\left(\omega_{2}\right)$, singly occupied by a fermion with spin-up [spin-down], $\left(\omega_{\uparrow[\downarrow]}\right)$, or empty $\left(\omega_{0}\right)$, we have that

$$
\begin{aligned}
& \omega_{2}=\left\langle\psi_{0}\left|\hat{n}_{j \uparrow} \hat{n}_{j \downarrow}\right| \psi_{0}\right\rangle, \\
& \omega_{\uparrow}=\left\langle\psi_{0}\left|\hat{n}_{j \uparrow}\right| \psi_{0}\right\rangle-\omega_{2}=\frac{n}{2}+m-\omega_{2}, \\
& \omega_{\downarrow}=\left\langle\psi_{0}\left|\hat{n}_{j \downarrow}\right| \psi_{0}\right\rangle-\omega_{2}=\frac{n}{2}-m-\omega_{2}, \\
& \omega_{0}=1-n+\omega_{2} .
\end{aligned}
$$

Here $\hat{n}_{j \sigma}=\hat{c}_{j \sigma}^{\dagger} \hat{c}_{j \sigma}$ is the number operator that checks site $j$ for a fermion of spin $\sigma=\uparrow, \downarrow, n=\left\langle\psi_{0}\left|\hat{n}_{j \uparrow}+\hat{n}_{j \downarrow}\right| \psi_{0}\right\rangle$ is the average ground state occupation number, and $m=(1 / 2)\left\langle\psi_{0}\left|\hat{n}_{j \uparrow}-\hat{n}_{j \downarrow}\right| \psi_{0}\right\rangle$ is the ground state magnetization per site. It follows that

$$
\rho_{j}=\sum_{\alpha=0, \uparrow, \downarrow} \omega_{\alpha}|\alpha\rangle_{j}\left\langle\left.\alpha\right|_{j}+\omega_{2} \mid \uparrow \downarrow\right\rangle_{j}\left\langle\left.\uparrow \downarrow\right|_{j} .\right.
$$

Combining Eqs. (1), (2), and (3) the single-site entanglement can then be expressed as

$$
\begin{aligned}
& \mathcal{E}=-\left(\frac{n}{2}+m-\omega_{2}\right) \log _{2}\left(\frac{n}{2}+m-\omega_{2}\right)- \\
& -\left(\frac{n}{2}-m-\omega_{2}\right) \log _{2}\left(\frac{n}{2}-m-\omega_{2}\right)- \\
& -\omega_{2} \log _{2} \omega_{2}-\left(1-n+\omega_{2)} \log _{2}\left(1-n+\omega_{2}\right) .\right.
\end{aligned}
$$

Suppose that the single-site entanglement thus constructed is non-analytic, as signaled by a singularity in its $(k-1)$ st derivative at a value, call it $g_{c}$, of some control parameter $g$ (with all lower-order derivatives being continuous and finite). To single out $g$ we write the Hamiltonian $\mathcal{H}(g)$ of the system as $\mathcal{H}(g)=\mathcal{H}_{0}+g \Lambda$ (with $\Lambda$ the conjugate operator to which $g$ couples, and with all other control parameters kept fixed and absorbed in $\mathcal{H}_{0}$ ). To be specific, we shall assume that $g$ is a magnetic field strength $(g=h)$, a chemical potential $(g=\mu)$, or a local on-site interaction $(g=U)$. Note that $g$ is precisely the field conjugate to one of the order parameters $\mathcal{O}_{g} \equiv\left\langle\psi_{0}|\Lambda| \psi_{0}\right\rangle$ that parameterize the reduced density matrix: the magnetization per site $m$, the average occupation number $n$, or the expectation value $\omega_{2}$ for double occupancy*.

Repeated differentiation of Eq. (4) yields

$$
\begin{aligned}
& \frac{\partial^{k-1} \mathcal{E}}{\partial g^{k-1}}=-\left(\frac{\partial^{k-1}}{\partial g^{k-1}}\left[\frac{n}{2}+m-\omega_{2}\right]\right) \log _{2}\left(\frac{n}{2}+m-\omega_{2}\right)- \\
& -\left(\frac{\partial^{k-1}}{\partial g^{k-1}}\left[\frac{n}{2}-m-\omega_{2}\right]\right) \log _{2}\left(\frac{n}{2}-m-\omega_{2}\right)-\frac{\partial^{k-1} \omega_{2}}{\partial g^{k-1}} \log _{2}\left(\omega_{2}\right)+ \\
& +\left(\frac{\partial^{k-1}}{\partial g^{k-1}}\left[n-\omega_{2}\right]\right) \log _{2}\left(1-n+\omega_{2}\right)+\ldots
\end{aligned}
$$

where $« \ldots . . »$ denote terms that contain lower-order derivatives, all of which are continuous and finite (since otherwise the $(k-2)$ nd derivative of $\mathcal{E}$ would also be singular, contrary to the assumption). A singularity in $\partial^{k-1} \mathcal{E} / \partial g^{k-1}$ must hence reside in the terms in containing derivatives of order $k-1$. Since the order parameter $\mathcal{O}_{g}$ conjugate to $g$ is one of the parameters $m, n$ or $\omega_{2}$ (or possibly a linear combination of $m, n$ and $\omega_{2}$ ), it follows that $\partial^{k-1} \mathcal{O}_{g} / \partial g^{k-1}$ also has a divergence or a discontinuity at $g=g_{c}$. By the

* The condition on $\mathcal{O}_{g}$ is much less restrictive than maybe first appears to be the case. In fact, a generic QPT in an interacting fermion system is precisely driven by a change of magnetic field, chemical potential, or a local (screened) interaction (in turn controlled by a change of the external pressure on the system, or by an applied voltage). 
Hellman-Feynman theorem, $\left\langle\psi_{0}|\partial \mathcal{H} / \partial g| \psi_{0}\right\rangle=\partial e_{0} / \partial g$, we conclude that the ground state energy $e_{0}$ has a singularity in its $k$ th derivative. But this is precisely what we mean by a $k$ th order QPT! Summarizing: a singularity in the $(k-1)$ st derivative of the single-site entanglement implies a $k$ th order QPT (with the proviso that the QPT is «generic», that is, driven by a change in magnetic field, chemical potential, or a local interaction) [31].

A few comments may here be in order. First, one could think that the close link between the scaling of $\partial^{k-1} \mathcal{E} / \partial g^{k-1}$ and that of $\partial^{k-1} \mathcal{O}_{g} / \partial g^{k-1}$ would allow for the critical exponent that controls $\mathcal{O}_{g}$ to be directly read off from $\partial^{k-1} \mathcal{E} / \partial g^{k-1}$. This inference is invalid, though. As a counter example, take a second order QPT $(k=2)$ with $\mathcal{O}_{g}=\omega_{2}$, where $\partial \omega_{2} / \partial u \sim\left|u-u_{c}\right|^{\rho-1} \rightarrow \infty$ as $g \rightarrow g_{c}=u_{c}$. By inspection of Eq. (5) one notes that the leading scaling of $\partial \mathcal{E} / \partial g$ will be governed by the same exponent $\rho$ only if $m$ and $n$ are independent of $\omega_{2}$, or, they depend on $\omega_{2}$ as a power with exponent $\geq 1$. Whether this is the case can only be determined on a case-to-case basis. A second, important comment concerns the logarithmic factors in (5). These will cause logarithmic divergences if one or several of the occupation parameters $\omega_{0}, \omega_{\uparrow}, \omega_{\downarrow}$ and $\omega_{2}$ vanish at the transition (cf. the expression in (2)). Such logarithmic corrections, multiplying the leading scaling of $\partial^{k-1} \mathcal{E} / \partial g^{k-1}$ inherited from $\mathcal{O}_{g}$, thus signal a change of the dimension of the accessible local Hilbert space as the system undergoes the transition. This is a useful and important property of the single-site entanglement scaling not shared by the scaling of $\mathcal{O}_{g}$ or its derivatives. It is here important to note that a spurious signaling of a $k$ th order QPT by a divergence in $\partial^{k-1} \mathcal{E} / \partial g^{k-1}$ caused by a vanishing occupation parameter is blocked by all lower-order derivatives of $\mathcal{E}$ being continuous and finite.

Putting our result to use, are we sure to identify all fermionic QPTs? In other words, is the non-analyticity in the single-site entanglement not only a sufficient, but also a necessary condition for the appearance of a QPT? The answer comes with a negative signature. First, the diagnostics becomes fuzzy for a QPT of infinite order [33], a Berezinskii-Kosterlitz-Thouless (BKT) type transition being the paradigm case [34]. Although the essential singularity that here is present in the ground state is expected to «infect» also the single-site entanglement via the reduced density matrix, its identification may be too difficult to serve as a useful tool. The situation becomes even less transparent for other non-conventional QPTs that are not associated with non-analyticities in the ground state energy [35], the transition a between two quantum Hall plateaus in the integer quantum Hall effect being an outstanding example. While our result does not apply to these cases, it is conceivable, maybe even expected, that the change of ground state at the transition will still show up as a non-analyticity in the entanglement. If and how this happens is a question well worth further studies. A class of QPTs where the single-site entanglement obviously fails as a marker for a QPT are those where the control parameter does not couple to a single-site. As we shall discuss below, while such QPTs in principle can be analyzed along the same lines as their simpler counterparts discussed above, the practical implementation of the analysis may meet with certain difficulties. There is, however, another, more insidious way that the single-site entanglement will fail to signal a QPT. This happens if all local basis states $|n\rangle_{j}=|0\rangle_{j},|\uparrow\rangle_{j},|\downarrow\rangle_{j}$, and $|\uparrow \downarrow\rangle_{j}$ become equally populated as one approaches the transition. As seen from (5), the $(k-1)$ st derivative terms then vanish identically, killing off the signal of the QPT. The simultaneous vanishing of $\partial \mathcal{E} / \partial g$ implies that $\mathcal{E}$ has a local extremum at the transition (expected to be a maximum since in this case all local basis states are equally represented in the make-up of the many-particle ground state). However, one cannot a priori exclude that $\mathcal{E}$ is at an extremum without the occurrence of a QPT. Hence, an extremum of the single-site entanglement does not necessarily signal a QPT. Whether a QPT is present or not in this case requires information beyond that provided by the entanglement measure. Unfortunately, this simple observation has been overlooked in some of the literature on the subject, leading to unnecessary confusion and speculations.

Having uncovered the general features of entanglement scaling at a fermionic QPT, let us now look at a few examples $[31,36,37]$.

\section{Case studies}

As our first example we take the 1D Hubbard model

$$
\mathcal{H}=-t \sum_{\substack{j=1 \\ \delta= \pm 1}}^{L} c_{j \alpha}^{\dagger} c_{j+\delta \alpha}+U \sum_{j=1}^{L} \hat{n}_{j \uparrow} \hat{n}_{j \downarrow}
$$

Here $c_{j \alpha}^{\dagger} c_{j \alpha}$ are the usual fermionic creation and annihilation operators attached to site $j$ of the lattice, with spin $\alpha=\uparrow, \downarrow$, and with $\hat{n}_{j \alpha} \equiv c_{j \alpha}^{\dagger} c_{j \alpha}$ the corresponding number operator. In the following we shall work with dimensionless quantities $u \equiv U / 4 t$ and $h \equiv \mu_{B} H / t$, putting $t=1$ (of dimension energy), and assume periodic boundary conditions. This model, which has long served as a paradigm for strongly correlated electron systems [38], has received renewed attention due to its possible realization in 1D optical lattices with trapped ultra-cold gases of fermionic atoms [39]. The sign and the strength of the on-site interaction $U$ and the tunneling rate $t$ between neighboring minima of the lattice potential can here be chosen at will by tuning a Feshbach resonance, thus making possible a fully «controllable» fermionic system governed by the Hubbard Hamiltonian in Eq. (6). 
At half-filling of the lattice, $n=1$ (i.e. with on average one fermion/site), the model exhibits a QPT at $u=0$, separating a Mott insulating phase $(u>0)$ from a metallic phase $(u<0)$. The ground state energy density becomes non-analytic at the transition, but allows for an asymptotic power series expansion with all derivatives being finite and continuous [40]. The QPT is thus of infinite order, and can be shown to belong to the BKT universality class [41]. As found by Gu et al. [42], the single-site entanglement has a maximum at the transition, reflecting the equipartition of empty-, singly- and doubly occupied local states when $u=0$ (non-interacting fermions). This transition is thus special on two counts: it is of infinite order and it supports an equipartition of local states. This makes it an exceptional example of a fermionic QPT, where no information can be deduced from the single-site entanglement measure.

A metal-insulator transition can also be triggered when $u>0$ by connecting the system to a particle reservoir and tuning the chemical potential $g \equiv \mu$ (with $\mu$ a dimensionless chemical potential multiplied by the hopping amplitude $t=1$ ). The corresponding Hamiltonian is given by that in Eq. (6), with the added term

$$
\mathcal{H}_{\mu}=-\mu \sum_{j=1}^{L}\left(\hat{n}_{j \uparrow}+\hat{n}_{j \downarrow}\right) .
$$

It is here important to point out that provided that there is no interaction with the reservoir, a pure state entanglement measure is still applicable at zero temperature. For the case of repulsive on-site interaction, $u>0$, and with $n \leq 1$, the system exhibits two quantum critical points [43]: $\mu_{c 1}=-2$ and

$$
\mu_{c 2}=2-4 \int_{0}^{\infty} J_{1}(\omega)(\omega[1+\exp (2 \omega u)])^{-1} d \omega,
$$

with $J_{1}(\omega)$ a first-order Bessel function. Both transitions are second-order with diverging charge susceptibilities $\chi_{C i}=c(u)\left|\mu-\mu_{c i}\right|^{-1 / 2}, i=1,2$ in the limits $\mu \rightarrow \mu_{c 1+}$ (empty lattice transition) and $\mu \rightarrow \mu_{c 2-}$ (Mott transition), respectively (with $c(u)$ a positive $u$-dependent constant). To obtain the single-site entanglement $\mathcal{E}$ we make the observation that $\mathcal{H}_{\mu}$ conserves spin and particle number for fixed $\mu$, and that hence the expression for $\mathcal{E}$ in (4) remains valid. Recalling from the Lieb-Mattis theorem [44] that the ground state has zero spin (for any $n$ with $n L$ an even integer) we put $m=0$ in (4). Using the Hellman-Feynman theorem, the value of $\omega_{2}$ can be extracted from the ground state energy via the relation $\omega_{2}=\left(\partial E_{0} / \partial u\right) / 4 L$. Exploiting the Bethe Ansatz solution of the model [43], $E_{0}$ can be expressed via a $1 / u$ expansion [45]:

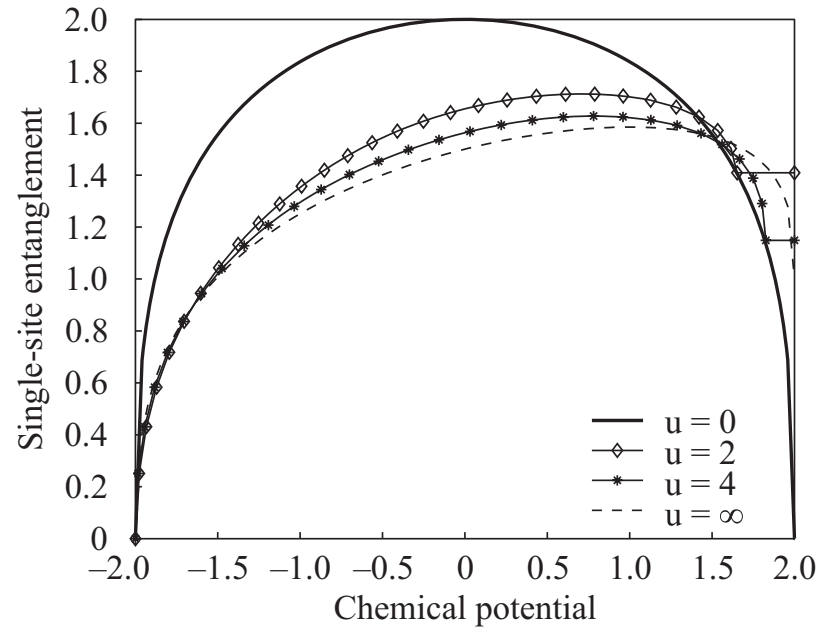

Fig. 1. Entanglement entropy $\mathcal{E}$ of a single site versus chemical potential $\mu$ for the repulsive Hubbard model. The plateaus correspond to half-filling $(n=1)$, cut off at $\mu=2$. The solid curve is that for free fermions $(u=0)$, plotted in the region $0 \leq n \leq 2$.

$$
\frac{E_{0}}{L}=-\frac{2}{\pi} \sin (\pi n)-\sum_{l=1}^{\infty} \kappa_{l}(n)\left(\frac{1}{4 u}\right)^{l} .
$$

The values of $\kappa_{l}(n)$ are tabulated to fifth order in Ref. 45 . The ground state energy in (8) also determines the chemical potential as function of filling: $\mu(n)=\partial E_{0} / \partial n$. By inverting $\mu(n)$ and inserting the resulting values for the $w$-parameters from (2) into (4) we can plot $\mathcal{E}$ vs. $\mu$ for any value of $u>1$. Some representative plots are shown in Fig. 1, together with the single-site entanglement for free fermions $(u=0)$.

In order to analytically explore the quantum critical regions $\mu \rightarrow \mu_{c 1+}$ and $\mu \rightarrow \mu_{c 2-}$ we first consider the $u \rightarrow \infty$ limit where $\omega_{2}=0$. In this limit (8) implies that $n(\mu)=(1 / \pi) \arccos (-\mu / 2)$. Combining this expression with (4) we obtain

$$
\frac{\partial \mathcal{E}}{\partial \mu}=(-1)^{i} \frac{\chi_{C i}}{2 \ln (2)}\left(\ln \left|\mu-\mu_{c i}\right|+\text { const }\right), i=1,2
$$

for $\mu \rightarrow \mu_{c 1+}$ and $\mu \rightarrow \mu_{c 2-}$, respectively. Turning to the case of large but finite $u$, we focus on the Mott transition $\mu \rightarrow \mu_{c 2-}$. A straightforward analysis, again using the Bethe Ansatz result in (8), yields for the leading behavior of the single-site entanglement:

$$
\frac{\partial \mathcal{E}}{\partial \mu}=-C(u) \chi_{C 2}
$$

with $C(u)$ a positive $u$-dependent constant.

The results in Eqs. (9) and (10) well illustrate our general discussion in the previous section. For finite $u$ the logarithms in Eq. (5) add up to the $u$-dependent constant $C(u)$, whereas in the limit $u \rightarrow \infty$ the entanglement 
measure detects a change in the dimension of the local Hilbert space, as signaled by the logarithmic correction to the leading scaling. In the $u \rightarrow \infty$ limit the singly occupied [empty] local states get suppressed when $\mu \rightarrow \mu_{c 1+}\left[\mu \rightarrow \mu_{c 2-}\right]$, while for finite $u$ both the metallic $\left(\mu<\mu_{c 2}\right)$ and insulating $\left(\mu>\mu_{c 2}\right)$ ground states are superpositions of all four types of local states $|0\rangle_{j},|\uparrow\rangle_{j},|\downarrow\rangle_{j}$, and $|\uparrow \downarrow\rangle_{j}$.

Let us next study the case of a QPT driven by an applied magnetic field, again using a Bethe Ansatz approach to the 1D Hubbard model as work horse. The Hamiltonian is now written:

$$
\mathcal{H}=-t \sum_{\substack{j=1 \\ \delta= \pm 1}}^{L} c_{j \alpha}^{\dagger} c_{j+\delta \alpha}+U \sum_{j=1}^{L} n_{j \uparrow} n_{j \downarrow}-\mu_{B} H \sum_{j=1}^{L} S_{j}^{z} .
$$

Here $S^{z_{j}}=\left(n_{j \uparrow}-n_{j \downarrow}\right) / 2$ is a spin- $1 / 2$ operator attached to lattice site $j$. As before, we use dimensionless quantities: $u \equiv U / 4 t$ and $h \equiv \mu_{B} H / t$. Focusing on the limit $|u|>>1$ with $u<0$ (attractive interaction), and with $n=1$ (half-filling), we can again exploit the Hellman-Feynman theorem. Together with the known Bethe Ansatz result for the ground state energy [46],

$$
E_{0} / 4 L=u(1 / 2-m)-(1 / 2 \pi) \sin (2 \pi m)+\mathcal{O}(1 / u),
$$

we obtain

$$
\omega_{2}=\frac{1}{4 L} \frac{\partial E_{0}}{\partial u}=\frac{1}{2}-m+\mathcal{O}\left(1 / u^{2}\right) .
$$

Neglecting the $\mathcal{O}\left(1 / u^{2}\right)$ corrections it follows immediately from Eq. (4) that

$$
\mathcal{E}=-2 m \log _{2}(2 m)-(1-2 m) \log _{2}\left(\frac{1}{2}-m\right), h \geq 0 .
$$

The dependence of the magnetization on the applied field can also be derived from the ground state energy, and one finds

$$
m(h)= \begin{cases}0, & 0 \leq h<h_{c 1}, \\ \frac{1}{2 \pi} \arccos \left(-\left(u+\frac{h}{4}\right)\right), & h_{c 1} \leq h \leq h_{c 2}, \\ \frac{1}{2}, & h_{c 2}<h,\end{cases}
$$

with lower [upper] critical field* $h_{c 1}=4(|u|-1) \times$ $\times\left[h_{c 2}=4(|u|+1)\right]$. The single-site entanglement as a function of magnetic field, $\mathcal{E}=\mathcal{E}(h)$, can now be read off from (4) and (14). The result for the $|u|>>1$ limit is plotted in

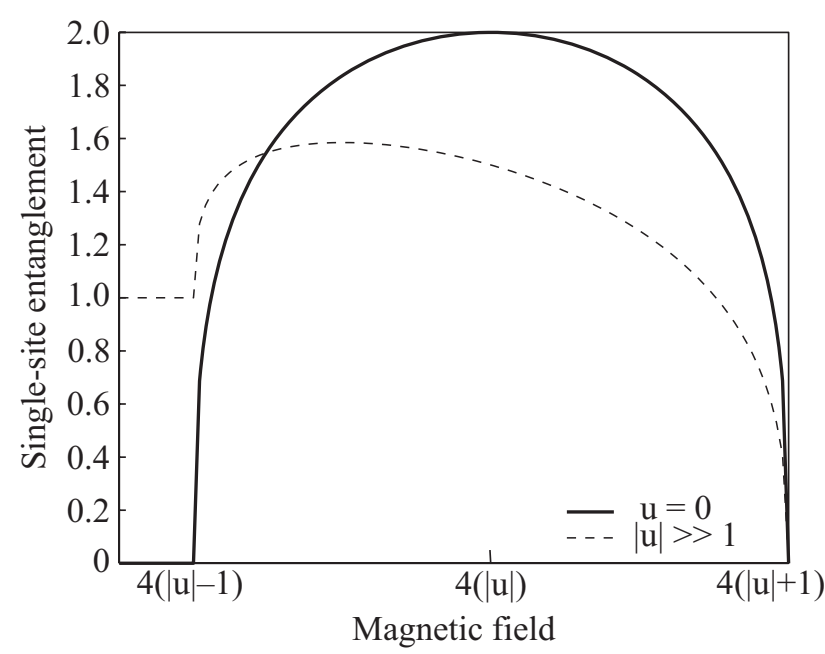

Fig. 2. Entanglement entropy $\mathcal{E}$ of a single site versus magnetic field $h$ for the attractive Hubbard model with $|u|>>1$ (dotted curve). For comparison, the single-site entanglement for the free case $(u=0)$ is shown by the solid curve (on a different scale).

Fig. 2 for large values of $h$. Note that in this limit there are two local states, $|0\rangle$ and $|\uparrow \downarrow\rangle$, available to the system when $h<h_{c 1}$, implying that $\mathcal{E}(h)=1$. In contrast, the fully magnetized state for $h>h_{c 2}$ is a direct product of local spin-up states, and hence $\mathcal{E}(h)=0$. For comparison we have plotted the single-site entanglement for free fermions also in Fig. 2 (for both positive and negative values of the magnetic field). This result is easily obtained from Ref. 46 by noting that $\omega_{2}=1 / 4-m^{2}$ when $u=0$, with $m=(1 / \pi) \arcsin (h / 4)$ in the interval $-4<h<4$.

The phase transitions at $h_{c 1}$ and $h_{c 2}$ are second order, with diverging spin susceptibilities $\chi_{S i}=$ $=\left(32 \pi^{2}\left|h-h_{c i}\right|\right)^{-1 / 2}, i=1,2$ [15]. The plot in (2) indeed suggests a corresponding divergence of $\partial \mathcal{E} / \partial h$ as $h \rightarrow h_{c 1+}$ and $h \rightarrow h_{c 2-}$ as predicted by our general result in the previous section. As an analytical check we write $u+h / 4=\left(h-h_{c i}\right) / 4+(-1)^{i}, i=1,2$ and expand $\partial \mathcal{E} / \partial h$ in $h-h_{c 1}$ and $h_{c 2}-h$, to obtain

$$
\frac{\partial \mathcal{E}}{\partial h}=(-1)^{i} \frac{\chi_{S i}}{\ln 2}\left(\ln \left|h-h_{c i}\right|+\text { const }\right), i=1,2
$$

for $h \rightarrow h_{c 1+}$ and $h \rightarrow h_{c 2^{-}}$, respectively. This confirms that $\partial \mathcal{E} / \partial h$ does diverge at the magnetic phase transitions, in accordance with our general result.

Turning to the half-filled case with repulsive interaction, $u>0$, a QPT now occurs only at the value of the field for which the magnetization saturates:

* The QPT at the upper critical field, the saturation point, is of a special type: Although being nontopological, there is no symmetry breaking at the transition. For a discussion, see [47]. 
$h_{c 2}=4\left(\sqrt{1+u^{2}}-u\right)[48]$. As shown by Takahashi, the ground state energy for any finite value of $u>0$ in the critical region $h \rightarrow h_{c 2-}$ can be expanded in terms of the expectation value for single spin-down occupancy [49]:

$$
\begin{aligned}
& \frac{E_{0}}{4 L}=-\left(\sqrt{1+u^{2}}-u\right)\left\langle n_{j \downarrow}\right\rangle_{0}+ \\
& +\frac{\pi^{2}}{24} \frac{1}{\sqrt{1+u^{2}}}\left\langle n_{j \downarrow}\right\rangle_{0}^{3}+\mathcal{O}\left(\left\langle n_{j \downarrow}\right\rangle_{0}^{4}\right) .
\end{aligned}
$$

With the same procedure as used for the attractive case above, Eq. (16), together with (2) and (4), yield:

$$
\frac{\partial \mathcal{E}}{\partial h}=\frac{C}{2 \ln 2} \chi_{S}\left(\ln \left|h-h_{c 2}\right|+\text { const }\right), h \rightarrow h_{c 2-} .
$$

Here $C=2-u / \sqrt{1+u^{2}}$, and $2 \pi \chi_{S}=\left(4+4 u^{2}\right)^{1 / 4} \times$ $\times\left|h-h_{c 2}\right|^{-1 / 2}$. The logarithmic correction in (17) now signals the suppression of all but the spin-up states as one approaches the saturation point $h_{c 2}$ from below.

The reason for the similarity of the scaling formulas in Eqs. (15) and (17) can be made transparent by exploiting a particle-hole transformation $\mathcal{T}$ for spin-up fermions:

$$
\mathcal{T}: c_{j \uparrow}^{\dagger} \leftrightarrow(-1)^{j} c_{j \uparrow},
$$

(leaving the spin-down fermions untouched). This transformation maps the zero-field repulsive Hubbard model with a chemical potential onto the half-filled attractive Hubbard model with an applied magnetic field. It follows that the single-site entanglement at $\mu_{c 1}\left(\mu_{c 2}\right)$ has the same behavior as at $h_{c 2}\left(h_{c 1}\right)$; cf. Figs. 3 and 4 .

As a second example, let us briefly discuss how the Mott-Hubbard transition in the 1D Hubbard model with long-range hopping gets signaled by the single-site entanglement. The model is defined by [50]

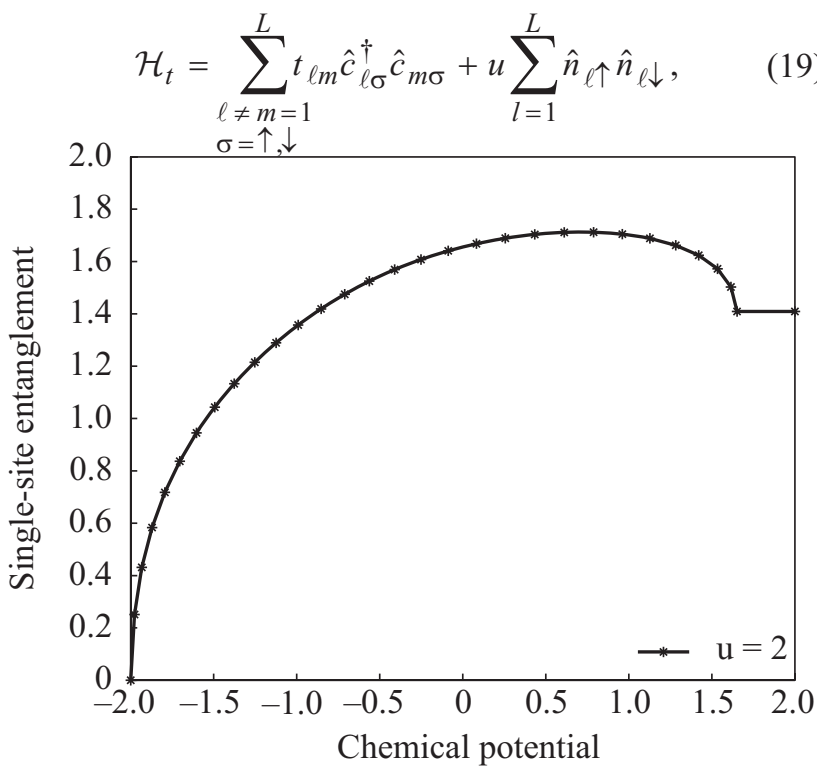

Fig. 3. Entanglement entropy $\mathcal{E}$ of a single site vs. chemical potential for $u=2$.

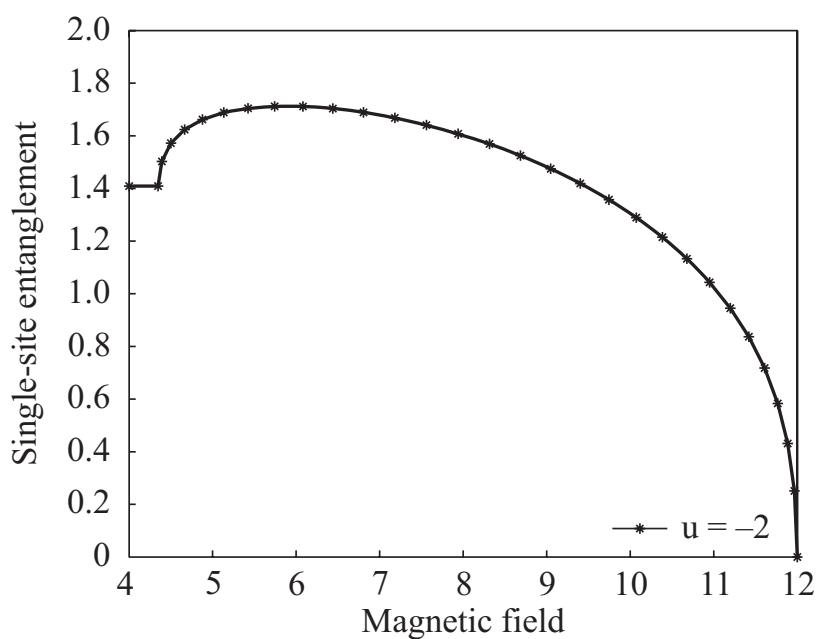

Fig. 4. Entanglement entropy $\mathcal{E}$ of a single site vs. magnetic field for $u=-2$ and at half-filling $(n=1)$, obtained from Fig. 3 via the particle-hole transformation $\mathcal{T}$ in Eq. (18).

with $t_{\ell m}=i(-1)^{(l-m)}(l-m)^{-1}$. The ground state energy density at half-filling is given by

$$
\begin{aligned}
& e_{0}=\left[u n-u_{c}(1-n) n\right) / 4-\left(1 /\left(24 u u_{c}\right)\right] \times \\
& \times\left[\left(u+u_{c}\right)^{3}-\left(\left(u+u_{c}\right)^{2}-4 u u_{c} n\right)^{3 / 2}\right]
\end{aligned}
$$

with $u_{c}=2 \pi$ the critical point [50]. This implies that $\omega_{2}=\partial e_{0} / \partial u$ has a discontinuity in its second order derivative with respect to $u$ at $u_{c}$ and hence the transition is third order. From Eq. (4) with $n=1$ it follows that the single site entanglement can be written as $\mathcal{E}=-\left(1-2 \omega_{2}\right) \log _{2}\left(1 / 2-\omega_{2}\right)-2 \omega_{2} \log _{2}\left(\omega_{2}\right)$ when no magnetic field is present (i.e. $m=0$ ), and one immediately verifies that $\partial^{2} \mathcal{E} / \partial u^{2}$ is also discontinuous at the transition point $u_{c}$. Since the local basis states do not become equally populated at $u_{c}$ - in contrast to the $u=0$ metal-insulator transition of the ordinary Hubbard model - the single-site entanglement here provides an accurate diagnostics of the transition. A plot of the single-site entanglement as function of $u$ is shown in Fig. 5. This QPT is unusual in exhibiting a discontinuity rather than a divergence in a higher-order derivative of the ground state energy. As one expects second- and higher-order phase transitions to be continuous one may worry that something is askew. However, the reason for the «anomaly» is simply that the Hamiltonian in [50] contains a long-range hopping process, thus breaking scale invariance at large distances (i.e. also in the «scaling limit»).

One can drive a Mott-Hubbard metal-insulator transition also by tuning the chemical potential when $u>u_{c}$, in exact analogy with the ordinary Hubbard model. Expressing $n$ as a function of $\mu$, and applying the Hellman-Feynman theorem to the ground state energy $e_{0}$ above, one obtains a discontinuity in $\partial n / \partial \mu$ at 


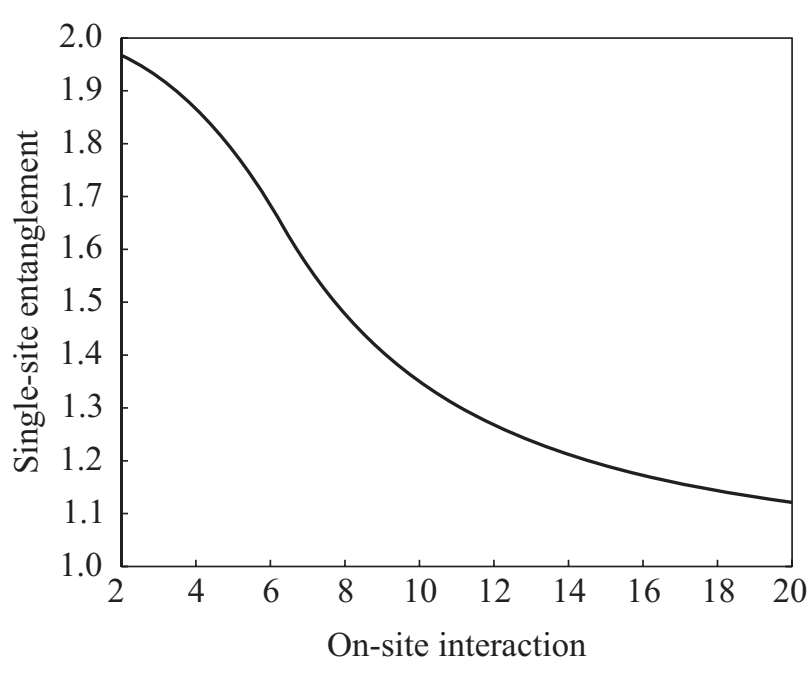

Fig. 5. Entanglement entropy $\mathcal{E}$ of a single site vs. on-site interaction $u$ in the Hubbard model with long-range hopping at half-filling $(n=1)$. Note that the Mott-Hubbard transition at $u_{c}=\pi / 2$ is away from the maximum of the entanglement entropy, in contrast to the case of the ordinary Hubbard model.

$\mu=\mu_{c}=\pi$ [51]. Eq. (5) immediately implies that $\partial \mathcal{E} / \partial \mu$ is also discontinuous at $\mu=\mu_{c}$, with the transition being second order. In the limit $u \rightarrow \infty$ this discontinuity is multiplied by a logarithmic divergent factor when $\mu \rightarrow \mu_{c-}$, reflecting the suppression of empty states in this case.

\section{Extensions of the theory: the one-dimensional $U V$ Hubbard model}

The modification of the ordinary one-dimensional Hubbard model obtained by allowing long-range hopping is only one of several possible ones. A more realistic extension of the model that mimics the effect of a finite-range Coulomb interaction is the 1D extended Hubbard model [52,53], alias the 1D UV Hubbard model. With the notation of Eq. (6), its Hamiltonian is written as

$$
\mathcal{H}_{U V}=-t \sum_{\substack{j=1 \\ \delta= \pm 1}}^{L} c_{j \alpha}^{\dagger} c_{j+\delta \alpha}+U \sum_{j=1}^{L} \hat{n}_{j \uparrow} \hat{n}_{j \downarrow}+V \sum_{j=1}^{L} \hat{n}_{j} \hat{n}_{j+1}
$$

where $\hat{n}_{j}=\hat{n}_{j \uparrow}+\hat{n}_{j \downarrow}$. The term $V$ is a nearest-neighbor interaction that emulates a finite-range (screened) Coulomb interaction. The inclusion of this term destroys the integrability of the model, but its possible phases can be extracted by exact diagonalization and variational techniques, supplemented by exact results in various limits. One finds a rich phase diagram in the $U V$-plane, with phases exhibiting enhanced correlations for charge- and spin density waves, singlet- and triplet superconducting correlations, and a region of phase separation $[53,54]$. Some of the coexistence lines separating the various phases were qualitatively reconstructed at half-filling by numerically identifying the ridges of the single-site entanglement as function of $U$ and $V$ [42]. Attempts to improve upon this result - including an identification of transitions to phases with enhanced pairing correlations - were subsequently carried out via a study of the scaling of the block entropy for the model [55].

Here we are more concerned with the detailed analyticity properties of the ground state entanglement as one approaches a QPT. As long as the QPT is driven by an external field that couples locally to single sites on the lattice, or by the on-site interaction $\sim U$, we can carry over the approach above intact.

As an example, let us consider the model in the $V \rightarrow \infty$ limit and at quarter filling $(n=1 / 2)$. For this case only every other site will be occupied since the energy cost to put two particles on neighboring sites is infinite. There are three distinct phases for this case: $U>4$, where every second site will occupied by precisely one particle; $-4<U<4$, with a mixture of doubly and singly occupied sites; and $U<-4$, where all sites are doubly occupied. As we tune the on-site interaction $U$, while staying in the $V \rightarrow \infty$ limit, we expect that the single-site entanglement will signal the QPTs that occur at $U=4$ (metal-insulator transition) and $U=-4$ (transition to a spin-gapped phase) respectively. To obtain an expression for the single-site entanglement, we take off from the parameterization of the reduced density matrix as given in Eq. (2). From Ref. 54 we have that

$$
\omega_{2}=(2 n-1)+\frac{1-n}{\pi} \arccos \left(-\frac{U}{4}\right),
$$

which at quarter filling $(n=1 / 2)$ takes the form

$$
\omega_{2}=\frac{1}{2 \pi} \arccos \left(-\frac{U}{4}\right) .
$$

The Lieb-Mattis theorem [44] implies that the ground state is a spin singlet $(m=0)$, and we thus conclude from Eqs. (2) and (22) that

$$
\begin{gathered}
\omega_{\uparrow}=\omega_{\downarrow}=\frac{1}{4}-\frac{1}{2 \pi} \arccos \left(-\frac{U}{4}\right), \\
\omega_{0}=\frac{1}{2}+\frac{1}{2 \pi} \arccos \left(-\frac{U}{4}\right) .
\end{gathered}
$$

Using Eq. (4) we can immediately write down the single-site entanglement:

$$
\begin{aligned}
& \mathcal{E}(U)=-\left(\frac{1}{2}-\frac{1}{\pi} \arccos \left(-\frac{U}{4}\right)\right) \log _{2}\left(\frac{1}{4}-\frac{1}{2 \pi} \arccos \left(-\frac{U}{4}\right)\right)- \\
& -\left(\frac{1}{2 \pi} \arccos \left(-\frac{U}{4}\right)\right) \log _{2}\left(\frac{1}{2 \pi} \arccos \left(-\frac{U}{4}\right)\right)- \\
& -\left(\frac{1}{2}+\frac{1}{2 \pi} \arccos \left(-\frac{U}{4}\right)\right) \log _{2}\left(\frac{1}{2}+\frac{1}{2 \pi} \arccos \left(-\frac{U}{4 \mu}\right)\right)=\dot{\mu}_{c} \stackrel{(25)}{=}
\end{aligned}
$$


Differentiating with respect to $U$ yields the following expression:

$$
\begin{aligned}
& \frac{\partial \mathcal{E}}{\partial U}=\frac{1}{8 \pi} \frac{1}{\sqrt{1-(U / 4)^{2}}}\left\{2 \log _{2}\left(\frac{1}{4}-\frac{1}{2 \pi} \arccos \left(-\frac{U}{4}\right)\right)-\right. \\
& \left.-\log _{2}\left(\frac{1}{2 \pi} \arccos \left(-\frac{U}{4}\right)\right)-\log _{2}\left(\frac{1}{2}+\frac{1}{2 \pi} \arccos \left(-\frac{U}{4}\right)\right)\right\} .
\end{aligned}
$$

By inspection of Eq. (26) we pinpoint divergences at $U= \pm 4$, signaling a second order QPT. Explicitly,

$$
\frac{\partial \mathcal{E}}{\partial U} \approx \frac{1}{4 \sqrt{2} \pi} \frac{1}{\sqrt{\left|U_{c}-U\right|}}\left\{\log _{2}\left(\left|U_{c}-U\right|\right)\right\}
$$

where we used the relations

$$
\begin{gathered}
1-(u / 4)^{2}=\left(U_{c}-U\right) / 2-\left(U_{c}-U\right) / 16 \approx\left(U_{c}-U\right) / 2 \\
\text { when } U_{c}=4,
\end{gathered}
$$

and

$$
\begin{gathered}
1-(U / 4)^{2}=\left(U-U_{c}\right) / 2-\left(U-U_{c}\right) / 16 \approx\left(U-U_{c}\right) / 2 \\
\text { when } U_{c}=-4 .
\end{gathered}
$$

The divergences in (27) were expected, since it is already known that $U= \pm 4$ define critical points for the two second-order QPTs: from a metal to an insulator at $U=4$, and from an ordinary metal to a metallic phase with superconducting correlations at $U=-4$ [54].

Suppose that we instead wish to explore a QPT driven by a change in the nearest-neighbor interaction $V$. The conjugate order-parameter is now that for nearest-neighbor occupancies, $\left\langle\psi_{0}\left|\hat{n}_{j} \hat{n}_{j+1}\right| \psi_{0}\right\rangle=n_{j} n_{j+1}$, which does not enter the reduced density matrix from which the single-site entanglement measure is constructed (cf. Eq. (2)). Hence, given our result from the previous section, the single-site entanglement is not expected to signal a QPT when driven by a change in $V$. The way out is obvious: We have to construct a two-site entanglement measure, based on the reduced density matrix for the two neighboring sites $j$ and $j+1$. (In analogy with the analysis above, which two sites that we choose is immaterial, provided that the system is translationally invariant.) The extension to a two-site measure is conceptually straightforward [55-58], allowing us in principle to carry over our results for non-analyticities in the entanglement unadorned. However, the practical implementation of the theory meets with certain obstacles. First, the reduced density matrix now acts on a 16-dimensional space, with many more entries to keep track on. Secondly, and more seriously, there are no exact analytical results for the ground state energy for arbitrary values of $U$ and $V$ that we can draw upon.
Fortunately, in the limit $U \rightarrow \infty$ (still at quarter filling, $n=1 / 2$ ), the theory simplifies and we can make some progress: When $U \rightarrow \infty$, double occupancies of single sites get suppressed, and as a consequence the dynamics becomes insensitive to the spin of the fermions. The local Hilbert spaces collapse to qubit spaces, spanned by the two states $|0\rangle$ («empty») and $|1\rangle$ («singly occupied»). Working with (dynamically) spinless fermions, one can then exploit the time-honored Jordan-Wigner transformation [59], and map the model onto the Bethe Ansatz solvable spin- $1 / 2 X X Z$ chain [60]

$$
\mathcal{H}=\sum_{i=1}^{\infty} \sigma_{i}^{x} \sigma_{i+1}^{x}+\sigma_{i}^{y} \sigma_{i+1}^{y}+\Delta \sigma_{i}^{z} \sigma_{i+1}^{z} .
$$

The Mott-Hubbard critical point at $V=2$ [59] in this way gets mapped onto the isotropic point $\Delta=1$, separating a spin liquid phase at $-1<\Delta<1$ from an Ising antiferromagnetic phase at $\Delta>1$. It follows that the desired information about the critical entanglement properties can be extracted by studying the two-site entanglement measure for the $X X Z$ model at $\Delta=1$. The two-site reduced density matrix for the $X X Z$ model is well-known [61-63], and the associated two-site entanglement is readily obtained [64], revealing a local maximum at $\Delta=1$, with all its higher-order derivatives being finite and continuous. This atypical behavior can be ascribed to the particular population of local states at the Mott transition which conspires to kill off the first-derivative of the two-site entanglement (with respect to $V$ ), in exact analogy to the vanishing of the first-derivative of the single-site entanglement when the local states are equally populated. This masks the expected non-analyticity of the entanglement, replacing it by a local maximum. In fact, the QPT is here of BKT type, just as for the Mott transition in the ordinary Hubbard model [41], making the detection of the non-analyticity (an essential singularity!) highly non-trivial.

As should be clear from this brief exposition, an analytical study of the complete phase diagram of the $U V$ extended Hubbard model from the perspective of entanglement scaling is not an easy task. Quite possibly it must await further theoretical breakthroughs.

\section{Outlook}

Before concluding, one may ask: «What is the advantage of probing the entanglement at a QPT rather than directly studying the behavior of the ground state energy?». There are several answers to this question. First, as we have already noted, certain QPTs can not be traced back to a nonanalyticity in the ground state energy, a case in point being the transitions between quantum Hall plateaus [35]. There are reasons to expect that a singular behavior of some entanglement measure may still apply to these transitions, and could serve as a convenient diag- 
nostic tool. To find out, one needs to practice on the simpler conventional QPTs, sorting out generic from accidental non-analyticities. Secondly, to understand to what extent non-local quantum correlations are implied in the scaling and universal properties exhibited by systems undergoing QPTs requires a detailed study of the associated entanglement properties. Our results for the Hubbard model [31,36,37], reviewed in the previous section, here provide a rich backdrop, with the entanglement scaling governed by the associated thermodynamic susceptibilities (and with logarithmic corrections encoding a change of dimension of the accessible local Hilbert spaces (see also Ref. 65)). Moreover, recent theoretical developments suggesting the use of many-particle entanglement for information processing may benefit from getting a firm handle on entanglement properties at QPTs. For example, certain schemes for quantum adiabatic computing [66] relies on (the assumed) entanglement scaling properties close to a QPT [67].

This is an area of research rich in opportunities. As it comes to models of interacting fermions, there is currently an ongoing activity by several research groups to explore QPTs in other extensions of the one-dimensional Hubbard model. Progress has been made for the bond-charge extended Hubbard model, where Anfossi et al. [56,57] have obtained important results, clarifying the role of multi-partite entanglement, as well as the relation between classical and quantum correlations at criticality More work should also be done - for fermionic and spin models - as it comes to unearthing effects from disorder, impurities, local fields, and boundaries. A particularly promising class of models to practice on - hitherto unexplored in this context - are the integrable multichain models discussed in the review by Zvyagin [68]. Another fascinating direction of research would be to study the quantum analogies $[69,70]$ of the elusive and highly nontrivial phase transitions «by breaking of analyticity», known from commensurate-incommensurate transitions $[71,72]$. A related class of phenomena in the context of low-dimensional quantum spin models have recently been discussed in Ref. 73. The study of entanglement behavior may here open up new landscapes for exploration.

We are grateful to K. Capelle and V.V. Franca for very useful comments. H.J. acknowledges financial support via a grant from the Swedish Research Council.

1. L. Amico, R. Fazio, A. Osterloh, and V. Vedral, quant-ph/0703044.

2. M.A. Nielsen and I.L. Chuang, Quantum Computation and Quantum Information, Cambridge University Press, Cambridge (2000).

3. E. Schrцdinger, Naturwissenschaften 23, 807 (1935).

4. J.S. Bell, Physics 1, 195 (1964).

5. A.Y. Kitaev, Ann. Phys. 303, 2 (2003).
6. R. Raussendorf and H.J. Briegel, Phys. Rev. Lett. 86, 5188 (2001).

7. A. Osterloh, L. Amico, G. Falci, and R. Fazio, Nature 416, 608 (2002).

8. T.J. Osborne and M.A. Nielsen, Phys. Rev. A66, 032110 (2002).

9. For a review, see S. Sachdev, Quantum Phase Transitions, Cambridge University Press, Cambridge (1999).

10. For a brief review, see P. Coleman and A.J. Schofield, $N a$ ture 433, 226 (2005).

11. S. Chakravarty, H.-Y. Kee, and К. Vцlker, Nature 428, 53 (2004).

12. M. Greiner, O. Mandel, T. Esslinger, T.W. Ндnsch, and I. Bloch, Nature 415, 39 (2002).

13. M.B. Plenio and S. Virmani, Quant. Inf. Comp. 7, 1 (2007).

14. L.-A. Wu, M.S. Sarandy, and D.A. Lidar, Phys. Rev. Lett. 93, 250404 (2004)

15. M.-F. Yang, Phys. Rev. A71, 030302 (2005).

16. L.-A. Wu, M.S. Sarandy, D.A. Lidar, and L.J. Sham, Phys. Rev. A74, 052335 (2006).

17. L.C. Venuti, C.D.E. Boschi, M. Roncaglia, and A. Scaramucci, Phys. Rev. A73, 010303 (2006).

18. F. Verstraete, M. Popp, and J.I. Cirac, Phys. Rev. Lett. 92, 027901 (2004).

19. Y. Chen, P. Zanardi, Z.D. Wang, and F.C. Zhang, New J. Phys. 8, 97 (2006).

20. T.R. de Oliveira, G. Rigolin, M.C. de Oliveira, and E. Miranda, Phys. Rev. Lett. 97, 170401 (2006).

21. G. Vidal, J.I. Latorre, E. Rico, and A. Kitaev, Phys. Rev. Lett. 90, 227902 (2003).

22. P. Calabrese and J. Cardy, J. Stat. Mech., P06002 (2004).

23. V.E. Korepin, Phys. Rev. Lett. 92, 096402 (2004).

24. K.M.R. Audenaert and M.B. Plenio, New J. Phys. 8, 266 (2006).

25. S. Bose, B.-Q. Lin, and V.E. Korepin, Phys. Rev. A72, $022345(2005)$.

26. L.C. Venuti, C.D.E. Boschi, and M. Roncaglia, quant-ph/0703202.

27. G. Ghirardi, L. Marinatto, and T. Weber, J. Stat. Phys. 108, 49 (2002).

28. P. Zanardi, Phys. Rev. A65, 042101 (2002).

29. W. K. Wootters, Phys. Rev. Lett. 80, 2245 (1998).

30. G. Vidal and R.F. Werner, Phys. Rev. A65, 032314 (2002).

31. D. Larsson and H. Johannesson, Phys. Rev. A73, 042320 (2006).

32. C.H. Bennett, H.J. Bernstein, S. Propescu, and B. Schumacher, Phys. Rev. A53, 2046 (1996).

33. C. Itoi and H. Mukaida, Phys. Rev. E60, 3688 (1999).

34. V.L. Berezinski1̄, Zh. Йksp. Teor. Fiz. 59, 907 (1970) [Sov. Phys. JETP 32, 493 (1971)]; J.M. Kosterlitz and D.J. Thouless, J. Phys. C6, 1181 (1973).

35. A. Kopp, X. Jia, and S. Chakravarty, cond-mat/0604152.

36. D. Larsson and H. Johannesson, Phys. Rev. Lett. 95, 196406 (2005).

37. D. Larsson and H. Johannesson, Phys. Rev. Lett. 96, 169906 (2006)

38. For a review, see F.H.L. Essler, H. Frahm, F. Gцhmann, A. Klsmper, and V. E. Korepin, The One-Dimensional Hub- 
bard Model, Cambridge University Press, Cambridge, (2005).

39. For a review, see M. Lewenstein, A. Sanpera, V. Ahufinger, B. Damski, A. Sen De, and U. Sen, cond-mat/0606771.

40. W. Metzner and D. Vollhardt, Phys. Rev. B39, 4462 (1989).

41. T. Giamarchi, Physica B230, 975 (1997).

42. S.-J. Gu, S.-S. Deng, Y.-Q. Lin, and H.-Q. Lin, Phys. Rev. Lett. 93, 086402 (2004).

43. E.H. Lieb and F.Y. Wu, Phys. Rev. Lett. 20, 1445 (1968); Physica A321, 1 (2003)

44. E. Lieb and D. Mattis, Phys. Rev. 125, 164 (1962).

45. J. Carmelo and D. Baeriswyl, Phys. Rev. B37, 7541 (1988).

46. C. Yang, A.N. Kocharian, and Y.L. Chiang, J. Phys. Condens. Mater. 12, 7433 (2000).

47. A. Fubini, T. Roscilde, V. Tognetti, M. Tusa, and P. Verrucchi, Eur. Phys. J. D38, 563 (2006).

48. M. Takahashi, Thermodynamics of One-Dimensional Solvable Models, Cambridge University Press, Cambridge, (1999).

49. M. Takahashi, Prog. Theor. Phys. 42, 1098 (1969).

50. F. Gebhard and A.E. Ruckenstein, Phys. Rev. Lett. 68, 244 (1992).

51. F. Gebhard, A. Girndt and A.E. Ruckenstein, Phys. Rev. B49, 10926 (1994).

52. A.A. Ovchinnikov, Sov. Phys. JETP 37, 176 (1973).

53. F. Mila and X. Zotos, Europhys. Lett. 24, 133 (1993).

54. K. Penc and F. Mila, Phys. Rev. B49, 9670 (1994).

55. S.-S. Deng, S.-J. Gu, and H.-Q. Lin, Phys. Rev. B74, 045103 (2006).
56. A. Anfossi, P. Giorda, A. Montorsi, and F. Traversa, Phys. Rev. Lett. 95, 056402 (2005).

57. A. Anfossi, P. Giorda, and A. Montorsi, Phys. Rev. B75, 165106 (2007).

58. Ц. Legeza and J. Solymon, Phys. Rev. Lett. 96, 116401 (2006).

59. T. Giamarchi, Quantum Physics in One Dimension, Oxford University Press (2004).

60. C.N. Yand and C.P. Yang, Phys. Rev. 150, 321, 327 (1966).

61. O.F. Syljuesen, Phys. Rev. A68, 060301 (2003).

62. S.-J. Gu, H.-Q. Lin, and Y.-Q. Li, Phys. Rev. A68, 042330 (2003).

63. S.-J. Gu, G.-S. Tian, and H.-Q. Lin, Phys. Rev. A71, $052322(2005)$.

64. S.-J. Gu, G.-S. Tian, and H.-Q. Lin, New J. Phys. 8, 61 (2006).

65. V.V. Franca and K. Capelle, Phys. Rev. A74, 042325 (2006).

66. E. Farhi, J. Goldstone, S. Gutmann, J. Lapan, A. Lundgren, and D. Preda, Science 292, 472 (2001).

67. J.I. Latorre and R. Orus, Phys. Rev. A69, 062302 (2004).

68. A.A. Zvyagin, J. Phys. A34, R21 (2001).

69. B. Hu and B. Li, Europhys. Lett. 46, 655 (1999).

70. X. Wang, H. Li, and B. Hu, Phys. Rev. A69, 054303 (2004).

71. S. Aubry, J. Phys. (Paris) 44, 147 (1983).

72. H. Johannesson, B. Schaub, and H. Suhl, Phys. Rev. B37, 9625 (1988)

73. A.A. Zvyagin, Phys. Rev. B72, 064419 (2005). 